Besonders hinzuweisen ist auch auf den Workshop „Software-Tools für die Einsatzplanung von Bürokommunikationssystemen". Hier soll gezeigt werden, wie Software-Programme den Organisator von Bürokommunikationsprojekten bei seiner Arbeit unterstützen können.
Eine weitere bemerkenswerte Komponente im Programm der ORGATECHNIK ist die Sonderschau "Neue Wege zum Wissen der Welt". In dieser Sonderschau werden verschiedene Zugangsformen zu Datenbanken wie BTX oder Datex-P vorgestellt.

\title{
Weitere Informationen:
}

Messe- und Ausstellungs-GmbH Köln

Messeplatz

Postfach 210760

5000 Köln 21 (Deutz)

Tel.: (02 21) 821-1

Btx: * 615021257 \#

\section{Frankfurter Buchmesse}

1. bis 6. Oktober

Messegelände Frankfurt

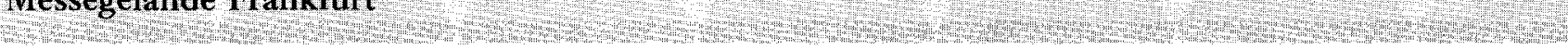

Die Frankfurter Buchmesse ist in erster Linie eine Messe für das gedruckte Buch. Sie bietet aber dieses Jahr im „Internationalen Zentrum für Bibliothekare und Dokumentare“ zusätzlich eine Anzahl von Informationsangeboten zu den „elektronischen Medien“, die nicht übersehen werden sollten. Auch wenn es sich nicht um juristische Applikationen handelt, vermitteln sie doch einen Eindruck vom Stand der Technik und veranschaulichen, wie entsprechende juristische Projekte aussehen könnten.

- Die Fa. Faxon (Amsterdam) führt das automatisierte Zeitschriftensystem „MicroLinx“, die Datenbank "Infoserv“ und die LoC-Serials auf CD-ROM vor.
- Das Programm „Freebase ${ }^{“}$ wird als Volltext-Retrieval-Konzept für CD-ROM präsentiert (Lüth-Systematik-Beratung, Dudenhofen).

- Der Springer-Verlag stellt CD-ROM-Applikationen im Bereich wissenschaftlicher Zeitschriften und das erste wissenschaftliche Software-Paket für Beilstein-Benutzer vor (SANDRA).

- Das Deutsche Institut für internationale pädagogische Forschung präsentiert in Verbindung mit dem Dokumentationsring Pädagogik einen Arbeitsplatz für die integrierte Recherche in internen und externen Datenbanken.

Von Interesse dürfte auch die Fachbuchausstellung „Datenverarbeitung: Theorie-Praxis-Information-Dokumentation" sein.

Weitere Informationen:

Ausstellungs- und Messe $\mathrm{GmbH}$

des Börsenvereins des Deutschen Buchhandels

Kleiner Hirschgraben 10/12

6000 Frankfurt am Main 